\title{
USAGE OF MAGNETIC IRON TO RAISE TOLERANCE OF SOME ORNAMENTAL TREES AND SHRUBS TO SOIL SALINITY IN CASE OF CHINA ROSE (HIBISCUS ROSA-SINENSIS L.)
}

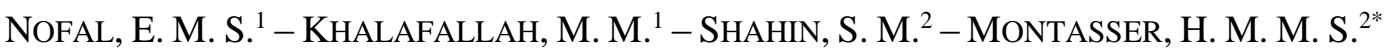 \\ ${ }^{l}$ Hort. Dept., Fac. Agric., Kafrelsheikh Univ., Kafr El Sheikh, Egypt \\ ${ }^{2}$ Botanical Gards. Res. Dept., Hort. Res. Inst., ARC, Giza, Egypt \\ *Corresponding author \\ e-mail: heba.montaser78@gmail.com
}

(Received $20^{\text {th }}$ Sep 2020; accepted $19^{\text {th }}$ Jan 2021)

\begin{abstract}
An investigation was conducted at Orman Botanical Garden, Giza, Egypt to reveal the role of magnetite at 2 or $4 \mathrm{~g} /$ pot in reducing the harmful effects of $\mathrm{NaCl}+\mathrm{CaCl}_{2}$ salt mixture added to the soil mixture at various concentrations, on growth and quality of Chinese hibiscus transplants. Interaction effect was also studied. The results showed that hibiscus transplants attained $100 \%$ survival, even at $8000 \mathrm{ppm}$ salinity level and the absence of magnetic iron. However, means of the different vegetative and root characteristics were progressively decreased as the concentration of salinity was increased scoring minimal values at the highest concentration. The opposite was true regarding magnetite treatments. A marked improvement in growth was also acquired by the different interaction treatments with superiority of combining between any level of salinity and the high rate of magnetite. The percentage of salt resistance index exhibited a descending decrement with salinity level increment, but it was, generally higher than $50 \%$ even at the high salinity level. A similar trend was obtained regarding leaf chemical composition, with few exceptions in both seasons. Accordingly, it is advised to apply magnetite at $4 \mathrm{~g} / \mathrm{pot}$ rates to saline soil for better growth of Hibiscus rosa-sinensis L. transplants.
\end{abstract}

Keywords: Hibiscus rosa-sinensis L., soil salinity, magnetic iron, chemical composition

\section{Introduction}

China Rose or Chinese hibiscus (Hibiscus rosa-sinensis L.) Fam. Malvaceae is one of the most beautiful, evergreen flowering shrubs used widely for landscape in Egypt. It is a large shrub, grow up to 5-7 m height, leaves usually simple ovate to $8-10 \mathrm{~cm}$ long, not lobed but toothed or nearly entire. It is native to subtropical and tropical areas as an ornamental plant for its glossy, flourish leaves and very large flowers that are born solitary in many colors on the leaf axils, for hedging and also glasshouses for summer bloom. It is easily propagated by cuttings, grafting and layering (Bailey, 1976).

Concerning its tolerance to salinity, Kratsch et al. (2008) categorized such ornamental shrub as sensitive to salinity, due to its tolerance of salts up to $650 \mathrm{ppm}$. On the other side, Mcfarland et al. (2014) found that it can tolerate soil salinity up to $2500 \mathrm{ppm}$, but can tolerate salinity of irrigation water up to $1250 \mathrm{ppm}$ only. So, it considered moderately sensitive to salts. Moreover, Khafagy et al. (2013) reported that it can tolerate the low level of diluted seawater (10\%), but higher levels (up to $40 \%$ ) gradually decreased all growth characters. That was documented by Ahmed (2017) who revealed that soil salinity higher than 2000 ppm clearly depressed it growth and flowering.

Many salinity disorders, such as plant growth reduction, delay flowering, decrease flower quality, tip and marginal leaf burn as a result of $\mathrm{Na}$ and $\mathrm{Cl}$ accumulation, and finally decreasing of aesthetical value of the plant were explored before by Mahmoud et al. (2008) on Dovyalis caffra and Lantana camara, Cassaniti et al. (2013) on 
Chrysanthemum morifolium, Dianthus cartophyllus, Gerberra jamesonii, Hippeastrum vittatum and Anthurium andreanum, Shahin et al. (2014) on tall fescue (Festuca arundinacea var. Festoria), El-Shewaikh et al. (2015) on areca (Dypsis lutescens), ElSayed et al. (2015) on saltbush (Atriplex halimus) and Shahin et al. (2017) on Casuarina equisetifolia and Eucalyptus rostrata. However, these disorders were corrected by some applicable ways elicited by Ahmed et al. (2011) on roselle (Hibiscus sabdariffa) Khafagy et al. (2013) and Ahmed (2017) on H. rosa-sinensis, Abdel-Fattah (2014) on Jacaranda acutifolia, Ahmed et al. (2016) on Acalypha wilkesiana and El-Sayed et al. (2019) on Enterolobium contortisiliquum.

The purpose of this trial was to evaluate the role of magnetite in alleviating injury of soil salinity on growth and aesthetical performance of Chinese hibiscus during rearing in the nursery.

\section{Materials and methods}

In order to find out the function of magnetic iron to mitigate damage of salinized soils on growth and flowering of China rose transplants an experiment was consummated under the full sun, temperature between $\left(27-38^{\circ} \mathrm{c}\right)$ the average percentage of humidity is $56 \%$ throughout the course of study at nursery of Orman Botanical Garden, Giza, Egypt during 2018 and 2019 consecutive seasons to determine the importance of this natural ore in enhancing growth of this plant under such stress.

Therefore, 4-months-old, uniform transplants of China rose of 17-18 cm height, carrying about 8-9 leaves were planted on April, $1^{\text {st }}$ for each season in 15-cm-diameter polyethylene black bags (one transplant/bag) filled with about $3.5 \mathrm{~kg}$ of sand and clay mixture at equal volume parts $(1: 1, \mathrm{v} / \mathrm{v})$. The physical and chemical properties of the soil mixture used in both seasons were measured and listed in Table 1.

Table 1. The physical and chemical analysis of the soil mixture used in 2018 and 2019 seasons

\begin{tabular}{|c|c|c|c|c|c|c|c|c|c|c|c|}
\hline Soil mixture & \multicolumn{4}{|c|}{ Particle size distribution (\%) } & \multirow{2}{*}{ S.P. } & \multirow{2}{*}{$\begin{array}{l}\text { E.C. } \\
\text { (dS/m) }\end{array}$} & \multirow{2}{*}{$\mathbf{p H}$} & \multicolumn{4}{|c|}{ Cations (meq/l) } \\
\hline \multirow{2}{*}{$\begin{array}{l}\text { Sand + Clay } \\
(1: 1, \mathrm{v} / \mathrm{v})\end{array}$} & Coarse sand & Fine sand & Silt & Clay & & & & $\mathrm{Ca}^{++}$ & $\mathrm{Mg}^{++}$ & $\mathrm{Na}^{+}$ & $\mathrm{K}^{+}$ \\
\hline & 38.6 & 31.3 & 20.6 & 9.5 & 25 & 6.5 & 7.86 & 17.8 & 14.2 & 33.1 & 0.9 \\
\hline \multirow{2}{*}{ Soil texture } & \multicolumn{4}{|c|}{ Anions (meq/l) } & \multicolumn{7}{|c|}{ Macro-and micro-elements (ppm) } \\
\hline & $\mathrm{HCO}_{3}^{-}$ & $\mathrm{Cl}^{-}$ & $\mathrm{SO}_{4}^{--}$ & $\mathrm{CO}_{3}$ & $\mathrm{~N}$ & $\mathrm{P}$ & $\mathrm{K}$ & $\mathrm{Fe}$ & $\mathrm{Zn}$ & $\mathrm{Mn}$ & $\mathrm{Cu}$ \\
\hline Sandy clay & 2.10 & 58.40 & 5.50 & 0.00 & 173.10 & 15.78 & 361.76 & 15.80 & 4.36 & 8.03 & 8.81 \\
\hline
\end{tabular}

Immediately before planting, the soil mixture was salinized with a mixture of $\mathrm{NaCl}$ and $\mathrm{CaCl}_{2}$ pure salts at equal weight parts $(1: 1, \mathrm{~g} / \mathrm{g})$ at the concentrations of 0,2000 , 4000, 6000 and $8000 \mathrm{ppm}$, while after planting the soil mixture was well mixed with magnetic iron ore $\left(\mathrm{Fe}_{3} \mathrm{O}_{4}, 22.5 \%\right)$ obtained from Alahram Mining Co., Maadi, Cairo at the rates of 0,2 and $4 \mathrm{~g} / \mathrm{plant}$, added as one batch at the commencing of the season. Salinity and magnetite treatments were connected factorially to create fifteen interactions.

During the course of the study, the plants under the different experimental treatments were fertilized 3 times with $2 \mathrm{~g} /$ plant of a compound chemical fertilizer (NPK + microelements i.e., Kristalon 19: 19: 19) and watered day by day. Besides, the various agricultural practices required for such plantation were carried out whenever needed. A factorial experiment based 
on a complete randomized design was accomplished in the two seasons, replicated thrice with five transplants for each replicate (Mead et al., 1993). The magnetite treatments represented the main factor and salinity levels represented the sub-factor.

At the end of each season (on October, $1^{\text {st }}$ ), the following data were recorded: survival $(\%)$, plant height $(\mathrm{cm})$, stem diameter $(\mathrm{cm})$, number of branches/plant, number of leaves/plant, leaf area $\left(\mathrm{cm}^{2}\right)$ using a planimeter, root length $(\mathrm{cm})$, aerial parts and roots fresh and dry weights $(\mathrm{g})$ after removing the soil under running water then lefts to dry and salt resistance index as a percentage (SRI \%) which was estimated from the equation of $\mathrm{Wu}$ and Huff (1983) as follows:

$$
\text { SRI }(\%)=\frac{\text { Mean root length of the longest root in salt treated plant }}{\text { mean root length of the longest root in control one }} \times 100
$$

Furthermore, the benefit coefficient of magnetite $\left(\mathrm{Fe}_{3} \mathrm{O}_{4}\right)$ under various salinity levels as a percentage (B. Coe \%) was calculated from the following equation:

$$
\text { B. Coe }(\%)=\frac{\text { Increase rate in dry matter of treated plant }}{\text { dry matter of control one }} \times 100
$$

In the second season only, concentrations of photosynthetic pigments (chlorophyll a, $\mathrm{b}$ and carotenoids, $\mathrm{mg} / \mathrm{g}$ f.w.) were assessed in fresh leaf samples using the method of Sumanta et al. (2014), whereas in dry ones, the percentages of nitrogen, phosphorus as well as potassium, sodium and chloride were measured according to the methods of Blake (1965), Luatanab and Olsen (1965) and Jackson (1973), consecutively. Proline concentration $(\mathrm{g} / 100 \mathrm{~g}$ d.w.) was evaluated in dry leaf sample using the method of Batels et al. (1973).

Data were statistically analyzed using the computer program of SAS Institute (2009) and Duncan's New Multiple Range to compare among means of treatments (Steel and Torrie, 1980).

\section{Results}

\section{Effect of soil salinity, magnetite and their interaction on vegetative and root growth parameters}

It is obvious from data presented in Table 2 that no mortality was occurred among transplants subjected to salinity concentration up to $8000 \mathrm{ppm}$, giving $100 \%$ survival $\%$ in the two seasons, although (Tables 3-6) means of plant height $(\mathrm{cm})$ stem diameter $(\mathrm{cm})$, No. branches and leaves / plant, leaf area $\left(\mathrm{cm}^{2}\right)$, root length $(\mathrm{cm})$ and aerial parts and roots fresh and dry weights $(\mathrm{g})$ were progressively declined with increasing salinity level to become the least at 8000 ppm concentration in both seasons. On the contrary, a gradual elevating in values of the previously stated traits was occurred as the rate of $\mathrm{Fe}_{3} \mathrm{O}_{4}$ was raised. Therefore, the utmost high means were attained by $4 \mathrm{~g} /$ pot rate in the two seasons. Interacting between salinity treatments and magnetite rates recorded also a marked improvement in averages of all vegetative and root growth characters mentioned above irrespective of salinity concentration. However, combining between salinity levels and $4 \mathrm{~g}$ magnetic iron/pot dose fulfilled higher records than salinity levels $+2 \mathrm{~g} /$ pot magnetite combination in most cases of both seasons. 
Table 2. Effect of salinity levels, magnetite rates and their interactions on survival and height of Hibiscus rosa-sinensis L. plant during 2018 and 2019 seasons

\begin{tabular}{|c|c|c|c|c|c|c|c|c|}
\hline \multirow{2}{*}{$\begin{array}{r}\mathrm{Fe}_{3} \mathrm{O}_{4} \text { rate } \\
(\mathrm{g} / \mathrm{plant}) \\
\text { Salinity level }(\mathrm{ppm})\end{array}$} & \multicolumn{4}{|c|}{ Survival (\%) } & \multicolumn{4}{|c|}{ Plant height $(\mathrm{cm})$} \\
\hline & 0.00 & $2 \mathrm{~g}$ & $4 \mathrm{~g}$ & Mean & 0.00 & $2 \mathrm{~g}$ & $4 \mathrm{~g}$ & Mean \\
\hline & \multicolumn{8}{|c|}{ First season 2018} \\
\hline 0.00 & $100.00 \mathrm{a}$ & $100.00 \mathrm{a}$ & $100.00 \mathrm{a}$ & $100.00 \mathrm{~A}$ & $37.00 \mathrm{~g}$ & $45.00 \mathrm{c}$ & $55.43 \mathrm{a}$ & $45.81 \mathrm{~A}$ \\
\hline 2000 & $100.00 \mathrm{a}$ & $100.00 \mathrm{a}$ & $100.00 \mathrm{a}$ & $100.00 \mathrm{~A}$ & $38.53 \mathrm{f}$ & $42.30 \mathrm{~d}$ & $47.07 \mathrm{~b}$ & $42.63 \mathrm{~B}$ \\
\hline 4000 & $100.00 \mathrm{a}$ & $100.00 \mathrm{a}$ & $100.00 \mathrm{a}$ & $100.00 \mathrm{~A}$ & $35.83 \mathrm{~h}$ & 39.30ef & $42.80 \mathrm{~d}$ & $39.31 \mathrm{C}$ \\
\hline 6000 & $100.00 \mathrm{a}$ & $100.00 \mathrm{a}$ & $100.00 \mathrm{a}$ & $100.00 \mathrm{~A}$ & $34.13 \mathrm{i}$ & $37.07 \mathrm{~g}$ & $40.17 \mathrm{e}$ & $37.12 \mathrm{D}$ \\
\hline 8000 & $100.00 \mathrm{a}$ & $100.00 \mathrm{a}$ & $100.00 \mathrm{a}$ & $100.00 \mathrm{~A}$ & $31.00 \mathrm{j}$ & $33.27 \mathrm{i}$ & $36.48 \mathrm{gh}$ & $33.58 \mathrm{E}$ \\
\hline \multirow[t]{2}{*}{ Mean } & $100.00 \mathrm{~A}$ & $100.00 \mathrm{~A}$ & $100.00 \mathrm{~A}$ & - & $35.30 \mathrm{C}$ & $39.39 \mathrm{~B}$ & $44.39 \mathrm{~A}$ & - \\
\hline & \multicolumn{8}{|c|}{ Second season 2019} \\
\hline 0.00 & $100.00 \mathrm{a}$ & $100.00 \mathrm{a}$ & $100.00 \mathrm{a}$ & $100.00 \mathrm{~A}$ & $36.33 d$ & $40.00 \mathrm{~b}$ & $47.07 \mathrm{a}$ & $41.13 \mathrm{~A}$ \\
\hline 2000 & $100.00 \mathrm{a}$ & $100.00 \mathrm{a}$ & $100.00 \mathrm{a}$ & $100.00 \mathrm{~A}$ & $32.10 \mathrm{~g}$ & $38.00 \mathrm{c}$ & $40.90 b$ & $37.00 \mathrm{~B}$ \\
\hline 4000 & $100.00 \mathrm{a}$ & $100.00 \mathrm{a}$ & $100.00 \mathrm{a}$ & $100.00 \mathrm{~A}$ & $29.87 \mathrm{~h}$ & $35.33 d$ & $37.53 \mathrm{c}$ & $34.24 \mathrm{C}$ \\
\hline 6000 & $100.00 \mathrm{a}$ & $100.00 \mathrm{a}$ & $100.00 \mathrm{a}$ & $100.00 \mathrm{~A}$ & $27.77 \mathrm{i}$ & $32.63 \mathrm{f}$ & $33.90 \mathrm{e}$ & $31.43 \mathrm{D}$ \\
\hline 8000 & $100.00 \mathrm{a}$ & $100.00 \mathrm{a}$ & $100.00 \mathrm{a}$ & $100.00 \mathrm{~A}$ & $25.67 \mathrm{j}$ & 28.87hi & $31.13 \mathrm{~g}$ & $28.56 \mathrm{E}$ \\
\hline Mean & $100.00 \mathrm{~A}$ & $100.00 \mathrm{~A}$ & $100.00 \mathrm{~A}$ & - & $30.35 \mathrm{C}$ & $34.97 \mathrm{~B}$ & $38.11 \mathrm{~A}$ & - \\
\hline
\end{tabular}

Mean followed by the same letter in a column or row do not differ significantly according to Duncan's new multiple range test

Table 3. Effect of salinity levels, magnetite rates and their interactions on stem diameter and No. branches/plant of Hibiscus rosa-sinensis L. plant during 2018 and 2019 seasons

\begin{tabular}{|c|c|c|c|c|c|c|c|c|}
\hline \multirow{2}{*}{$\begin{array}{r}\mathrm{Fe}_{3} \mathrm{O}_{4} \text { rate } \\
(\mathrm{g} / \mathrm{plant}) \\
\text { Salinity level }(\mathrm{ppm})\end{array}$} & \multicolumn{4}{|c|}{ Stem diameter $(\mathrm{cm})$} & \multicolumn{4}{|c|}{ No. branches/plant } \\
\hline & 0.00 & $2 \mathrm{~g}$ & $4 \mathrm{~g}$ & Mean & 0.00 & $2 \mathrm{~g}$ & $4 \mathrm{~g}$ & Mean \\
\hline & \multicolumn{8}{|c|}{ First season 2018} \\
\hline 0.00 & $0.500 \mathrm{fg}$ & $0.667 \mathrm{ab}$ & $0.700 \mathrm{a}$ & $0.622 \mathrm{~A}$ & $2.67 \mathrm{~cd}$ & $3.00 \mathrm{c}$ & $4.00 \mathrm{a}$ & $3.22 \mathrm{~A}$ \\
\hline 2000 & $0.467 \mathrm{~g}$ & $0.567 \mathrm{de}$ & $0.633 b c$ & $0.556 \mathrm{~B}$ & $2.67 \mathrm{~cd}$ & $3.00 \mathrm{c}$ & $3.50 \mathrm{~b}$ & $3.06 \mathrm{~B}$ \\
\hline 4000 & $0.600 \mathrm{~cd}$ & $0.600 \mathrm{~cd}$ & $0.600 \mathrm{~cd}$ & $0.600 \mathrm{~A}$ & $2.67 \mathrm{~cd}$ & $3.00 \mathrm{c}$ & $3.00 \mathrm{c}$ & $2.89 \mathrm{~B}$ \\
\hline 6000 & $0.533 \mathrm{ef}$ & $0.567 \mathrm{de}$ & $0.600 \mathrm{~cd}$ & $0.567 \mathrm{~B}$ & $2.33 \mathrm{de}$ & $2.67 \mathrm{~cd}$ & $2.67 \mathrm{~cd}$ & $2.56 \mathrm{C}$ \\
\hline 8000 & $0.533 \mathrm{ef}$ & $0.533 \mathrm{ef}$ & $0.567 \mathrm{de}$ & $0.544 \mathrm{~B}$ & $1.67 f$ & $1.67 \mathrm{f}$ & $2.00 \mathrm{ef}$ & $1.78 \mathrm{D}$ \\
\hline \multirow[t]{2}{*}{ Mean } & $0.427 \mathrm{C}$ & $0.587 \mathrm{~B}$ & $0.620 \mathrm{~A}$ & - & $2.40 \mathrm{C}$ & $2.67 \mathrm{~B}$ & $3.03 \mathrm{~A}$ & - \\
\hline & \multicolumn{8}{|c|}{ Second season 2019} \\
\hline 0.00 & $0.410 \mathrm{fg}$ & $0.557 b$ & $0.613 a$ & $0.527 \mathrm{~A}$ & $1.67 \mathrm{~g}$ & $2.33 \mathrm{e}$ & $2.67 \mathrm{~cd}$ & $2.22 \mathrm{C}$ \\
\hline 2000 & $0.387 \mathrm{~g}$ & $0.463 \mathrm{de}$ & $0.513 \mathrm{c}$ & $0.454 \mathrm{BC}$ & $2.00 \mathrm{f}$ & $3.00 \mathrm{~b}$ & $3.33 \mathrm{a}$ & $2.78 \mathrm{~A}$ \\
\hline 4000 & $0.470 \mathrm{de}$ & $0.477 \mathrm{~cd}$ & $0.467 \mathrm{de}$ & $0.471 \mathrm{~B}$ & $1.67 \mathrm{~g}$ & $2.83 b c$ & $3.00 \mathrm{~b}$ & $2.50 \mathrm{~B}$ \\
\hline 6000 & $0.437 \mathrm{~d}-\mathrm{f}$ & $0.433 \mathrm{ef}$ & $0.440 \mathrm{~d}-\mathrm{f}$ & $0.437 \mathrm{CD}$ & $1.63 \mathrm{~g}$ & $2.67 \mathrm{~cd}$ & $2.83 \mathrm{bc}$ & $2.38 \mathrm{BC}$ \\
\hline 8000 & $0.440 \mathrm{~d}-\mathrm{f}$ & $0.407 \mathrm{fg}$ & $0.417 f g$ & $0.421 \mathrm{D}$ & $1.27 \mathrm{~h}$ & $1.83 \mathrm{fg}$ & $2.44 \mathrm{de}$ & $1.84 \mathrm{D}$ \\
\hline Mean & $0.429 \mathrm{C}$ & $0.467 \mathrm{~B}$ & $0.490 \mathrm{~A}$ & - & $1.65 \mathrm{C}$ & $2.53 \mathrm{~B}$ & $2.85 \mathrm{~A}$ & - \\
\hline
\end{tabular}

Mean followed by the same letter in a column or row do not differ significantly according to Duncan's new multiple range test 
Table 4. Effect of salinity levels, magnetite rates and their interactions on leaf and root length and root callar diameter of Hibiscus rosa-sinensis L. plant during 2018 and 2019 seasons

\begin{tabular}{|c|c|c|c|c|c|c|c|c|c|c|c|c|}
\hline \multirow{2}{*}{$\begin{array}{r}\mathrm{Fe}_{3} \mathrm{O}_{4} \text { rate } \\
\text { (g/plant) } \\
\text { Salinity level (ppm) }\end{array}$} & \multicolumn{4}{|c|}{ No. leaves/plant } & \multicolumn{4}{|c|}{ Leaf area $\left(\mathrm{cm}^{2}\right)$} & \multicolumn{4}{|c|}{ Root length $(\mathrm{cm})$} \\
\hline & 0.00 & $2 \mathrm{~g}$ & $4 \mathrm{~g}$ & Mean & 0.00 & $2 \mathrm{~g}$ & $4 \mathrm{~g}$ & Mean & 0.00 & $2 \mathrm{~g}$ & $4 \mathrm{~g}$ & Mean \\
\hline & \multicolumn{12}{|c|}{ First season 2018} \\
\hline 0.00 & $33.67 \mathrm{e}$ & $42.33 b$ & $48.67 \mathrm{a}$ & $41.56 \mathrm{~A}$ & $28.40 \mathrm{~cd}$ & $30.77 \mathrm{~b}$ & $33.57 \mathrm{a}$ & $30.91 \mathrm{~A}$ & $33.33 \mathrm{e}$ & $36.40 \mathrm{c}$ & $40.67 \mathrm{a}$ & $36.80 \mathrm{~A}$ \\
\hline 2000 & $32.67 \mathrm{e}$ & $39.37 \mathrm{c}$ & $42.03 \mathrm{~b}$ & 38.02B & $27.40 \mathrm{de}$ & $29.53 c$ & $33.23 \mathrm{a}$ & $30.06 \mathrm{~B}$ & $32.03 \mathrm{e}$ & $35.00 \mathrm{~d}$ & $38.50 \mathrm{~b}$ & $35.18 \mathrm{~B}$ \\
\hline 4000 & $29.07 \mathrm{f}$ & $34.43 \mathrm{e}$ & $37.10 \mathrm{~d}$ & $33.53 \mathrm{C}$ & $25.37 f$ & $27.20 \mathrm{de}$ & $30.97 \mathrm{~b}$ & $27.84 \mathrm{C}$ & $27.77 \mathrm{~g}$ & $30.63 \mathrm{f}$ & $32.87 \mathrm{e}$ & $30.42 \mathrm{C}$ \\
\hline 6000 & $24.77 \mathrm{~g}$ & $29.03 f$ & $34.27 f$ & 29.36D & $22.50 \mathrm{~h}$ & $23.97 \mathrm{~g}$ & $26.90 \mathrm{e}$ & $24.46 \mathrm{D}$ & $24.37 \mathrm{i}$ & $26.40 \mathrm{~h}$ & $28.60 \mathrm{~g}$ & $26.46 \mathrm{D}$ \\
\hline 8000 & $20.67 \mathrm{~h}$ & $23.03 \mathrm{~g}$ & $30.17 \mathrm{f}$ & $24.62 \mathrm{E}$ & $20.00 \mathrm{i}$ & $21.70 \mathrm{~h}$ & $24.17 f g$ & $21.96 \mathrm{E}$ & $19.70 \mathrm{k}$ & $21.60 \mathrm{j}$ & $23.43 \mathrm{i}$ & $21.58 \mathrm{E}$ \\
\hline \multirow[t]{2}{*}{ Mean } & $28.17 \mathrm{C}$ & 33.64B & $38.45 \mathrm{~A}$ & - & $24.73 \mathrm{C}$ & 26.63B & 29.77A & - & $27.44 \mathrm{C}$ & 30.01B & $32.81 \mathrm{~A}$ & - \\
\hline & \multicolumn{12}{|c|}{ Second season 2019} \\
\hline 0.00 & $32.40 \mathrm{~cd}$ & $36.60 \mathrm{~b}$ & $40.87 \mathrm{a}$ & $36.62 \mathrm{~A}$ & $33.00 \mathrm{~d}$ & $39.43 b$ & $41.60 \mathrm{a}$ & $38.01 \mathrm{~A}$ & $19.33 \mathrm{~h}$ & 22.73de & $31.00 \mathrm{a}$ & $24.36 \mathrm{~A}$ \\
\hline 2000 & $29.10 \mathrm{e}$ & $33.80 \mathrm{c}$ & $35.47 \mathrm{~b}$ & $32.79 \mathrm{~B}$ & $29.47 \mathrm{f}$ & $32.80 \mathrm{~d}$ & $37.27 \mathrm{c}$ & $33.18 \mathrm{~B}$ & $17.40 \mathrm{ij}$ & $21.20 \mathrm{fg}$ & $27.07 \mathrm{~b}$ & $21.89 \mathrm{~B}$ \\
\hline 4000 & $26.43 \mathrm{f}$ & $31.33 \mathrm{~d}$ & $32.50 \mathrm{~cd}$ & $30.09 \mathrm{C}$ & $24.43 \mathrm{ij}$ & $28.90 f$ & $33.20 \mathrm{~d}$ & $28.84 \mathrm{C}$ & $16.80 \mathrm{j}$ & $20.10 \mathrm{gh}$ & $25.50 \mathrm{c}$ & $20.80 \mathrm{C}$ \\
\hline 6000 & $24.47 \mathrm{~g}$ & $27.93 \mathrm{e}$ & $29.17 \mathrm{e}$ & 27.19D & $23.93 \mathrm{j}$ & $27.00 \mathrm{gh}$ & $30.93 \mathrm{e}$ & $27.29 \mathrm{D}$ & $16.10 \mathrm{jk}$ & $19.27 \mathrm{~h}$ & $23.77 \mathrm{~d}$ & 19.71D \\
\hline 8000 & $21.83 \mathrm{~h}$ & $24.83 \mathrm{~g}$ & $26.47 f$ & $24.38 \mathrm{E}$ & $21.33 \mathrm{k}$ & $25.77 \mathrm{hi}$ & $28.10 \mathrm{fg}$ & $25.07 \mathrm{E}$ & $15.03 \mathrm{k}$ & $17.70 \mathrm{i}$ & $21.80 \mathrm{ef}$ & $18.18 \mathrm{E}$ \\
\hline Mean & $26.85 \mathrm{C}$ & $30.90 \mathrm{~B}$ & $32.89 \mathrm{~A}$ & - & $26.43 \mathrm{C}$ & 30.78B & $34.22 \mathrm{~A}$ & - & $17.60 \mathrm{C}$ & 20.20B & $25.83 \mathrm{~A}$ & - \\
\hline
\end{tabular}

Mean followed by the same letter in a column or row do not differ significantly according to Duncan's new multiple range test

Table 5. Effect of salinity levels, magnetite rates and their interactions on aerial parts fresh and dry weights of Hibiscus rosa-sinensis L. plant during 2018 and 2019 seasons

\begin{tabular}{|c|c|c|c|c|c|c|c|c|}
\hline \multirow{2}{*}{$\begin{array}{r}\mathrm{Fe}_{3} \mathrm{O}_{4} \text { rate } \\
\text { (g/plant) } \\
\text { Salinity level (ppm) }\end{array}$} & \multicolumn{4}{|c|}{ Aerial parts fresh weights (g) } & \multicolumn{4}{|c|}{ Aerial parts dry weights (g) } \\
\hline & 0.00 & $2 \mathrm{~g}$ & $4 \mathrm{~g}$ & Mean & 0.00 & $2 \mathrm{~g}$ & $4 \mathrm{~g}$ & Mean \\
\hline & \multicolumn{8}{|c|}{ First season 2018} \\
\hline 0.00 & $42.27 \mathrm{~d}$ & $47.10 \mathrm{~b}$ & $52.20 \mathrm{a}$ & $47.19 \mathrm{~A}$ & $12.40 \mathrm{~d}$ & $13.80 \mathrm{~b}$ & $17.43 \mathrm{a}$ & $15.54 \mathrm{~A}$ \\
\hline 2000 & $37.67 \mathrm{e}$ & $41.53 \mathrm{~d}$ & $44.33 \mathrm{c}$ & 41.18B & $10.76 \mathrm{~d}$ & $12.18 \mathrm{c}$ & $14.37 \mathrm{~b}$ & 12.44B \\
\hline 4000 & $35.10 \mathrm{~g}$ & $34.60 f$ & $38.70 \mathrm{e}$ & $36.13 \mathrm{C}$ & $9.70 \mathrm{e}$ & 10.10de & $12.90 \mathrm{c}$ & $10.90 \mathrm{C}$ \\
\hline 6000 & $26.90 \mathrm{~h}$ & $28.43 \mathrm{~h}$ & $32.07 \mathrm{~g}$ & 29.13D & $7.50 \mathrm{~g}$ & $8.37 \mathrm{f}$ & $10.43 \mathrm{de}$ & $8.77 \mathrm{D}$ \\
\hline 8000 & $20.57 j$ & $21.03 \mathrm{j}$ & $24.00 \mathrm{i}$ & $21.87 \mathrm{E}$ & $5.43 \mathrm{~h}$ & $6.07 \mathrm{~h}$ & $8.00 \mathrm{fg}$ & $6.50 \mathrm{E}$ \\
\hline \multirow[t]{2}{*}{ Mean } & $32.50 \mathrm{C}$ & 34.54B & $38.26 \mathrm{~A}$ & - & $9.16 \mathrm{C}$ & $10.10 \mathrm{~B}$ & $12.63 \mathrm{~A}$ & - \\
\hline & \multicolumn{8}{|c|}{ Second season 2019} \\
\hline 0.00 & $23.97 \mathrm{~d}$ & $25.93 \mathrm{c}$ & $32.17 \mathrm{a}$ & $27.36 \mathrm{~A}$ & $8.80 \mathrm{ef}$ & $9.87 \mathrm{~cd}$ & $12.67 \mathrm{a}$ & $10.44 \mathrm{~A}$ \\
\hline 2000 & $21.53 \mathrm{e}$ & $24.07 \mathrm{~d}$ & $27.87 \mathrm{~b}$ & 24.49B & $8.13 \mathrm{fg}$ & $9.27 \mathrm{de}$ & $11.63 b$ & $9.68 \mathrm{~B}$ \\
\hline 4000 & $19.40 \mathrm{f}$ & $21.80 \mathrm{e}$ & $25.17 \mathrm{~cd}$ & $22.12 \mathrm{C}$ & $7.14 \mathrm{~h}$ & $8.23 \mathrm{fg}$ & $10.53 c$ & $8.63 \mathrm{C}$ \\
\hline 6000 & $16.40 \mathrm{~g}$ & $19.17 \mathrm{f}$ & $22.10 \mathrm{e}$ & $19.22 \mathrm{D}$ & $5.80 \mathrm{jk}$ & 7.00hi & $9.30 \mathrm{e}$ & 7.37D \\
\hline 8000 & $13.83 \mathrm{~h}$ & $16.73 \mathrm{~g}$ & $19.87 f$ & $16.81 \mathrm{E}$ & $4.90 \mathrm{k}$ & $6.10 \mathrm{ij}$ & $7.63 \mathrm{gh}$ & $6.21 \mathrm{E}$ \\
\hline Mean & $19.03 \mathrm{C}$ & $21.54 \mathrm{~B}$ & $25.43 \mathrm{~A}$ & - & $6.95 \mathrm{C}$ & $8.09 \mathrm{~B}$ & $10.35 \mathrm{~A}$ & - \\
\hline
\end{tabular}

Mean followed by the same letter in a column or row do not differ significantly according to Duncan's new multiple range test

\section{Salt resistance index (SRI \%) and benefit coefficient of magnetite (B. coe. \%)}

As shown in Table 7, a progressive decrease in the percentages of salt resistance index was acquired due to the gradual increment in salinity concentration to reach the least values by $8000 \mathrm{ppm}$ level in the two seasons. However, these values were higher 
than $50 \%$ at such high concentration $(8000 \mathrm{ppm}) ; 58.78 \%$ in the first season and $75.14 \%$ in the second one pointing to the good tolerance of Chinese hibiscus to soil salinity up to $8000 \mathrm{ppm}$. On the other side, adding $\mathrm{Fe}_{3} \mathrm{O}_{4}$ was found to be out of order, especially at $4 \mathrm{~g} /$ pot rate, which gave means for such index closely near to those of control ones. The rates of $2 \mathrm{~g} / \mathrm{pot}$, however, slightly improved the percent of this index in the $1^{\text {st }}$ season, whilst in the second one, caused a significant increase in it. Moreover, combining between magnetite (especially at $4 \mathrm{~g} /$ pot dose) and various salinity levels was worthless compared to control treatment in the two seasons. Thus, application of $\mathrm{Fe}_{3} \mathrm{O}_{4}$ may be unvalued to improve resistance of Chinese hibiscus to soil salinity. This result may be unexpected and deserve more investigation to confirm it.

The opposite was the right concerning the benefit coefficient of magnetite percentages (Table 7) that cleared a great benefit for applying of $\mathrm{Fe}_{3} \mathrm{O}_{4}$ in production of dry matter, especially at the high salinity concentration $(8000 \mathrm{ppm})$ in the first season and at 4000, 6000 and 8000 ppm concentrations in the second one. In addition, drenching the soil mixture with magnetic iron at the rate of $4 \mathrm{~g} /$ pot attained the highest benefit for dry matter formation in both seasons, followed by $2 \mathrm{~g} / \mathrm{pot}$ rate relative to control treatment. Results of the combined treatments in the first season were fluctuated except for $8000 \mathrm{ppm}$ level + magnetite at any rate combination which showed higher benefit than other combinations. In the second season, all combinations cleared more advantage than control ones giving heaviest dry matter at all.

Table 6. Effect of salinity levels, magnetite rates and their interactions on roots fresh and dry weights of Hibiscus rosa-sinensis L. plant during 2018 and 2019 seasons

\begin{tabular}{|c|c|c|c|c|c|c|c|c|}
\hline \multirow{2}{*}{$\begin{array}{r}\mathrm{Fe}_{3} \mathrm{O}_{4} \text { rate } \\
(\mathrm{g} / \mathrm{plant}) \\
\text { Salinity level }(\mathrm{ppm})\end{array}$} & \multicolumn{4}{|c|}{ Roots fresh weights (g) } & \multicolumn{4}{|c|}{ Roots dry weights (g) } \\
\hline & 0.00 & $2 \mathrm{~g}$ & $4 \mathrm{~g}$ & Mean & 0.00 & $2 \mathrm{~g}$ & $4 \mathrm{~g}$ & Mean \\
\hline & \multicolumn{8}{|c|}{ First season 2018} \\
\hline 0.00 & $13.97 \mathrm{~d}$ & $16.80 \mathrm{~b}$ & $17.57 \mathrm{a}$ & $16.11 \mathrm{~A}$ & $6.20 \mathrm{ef}$ & $7.07 \mathrm{c}$ & $8.40 \mathrm{a}$ & $7.22 \mathrm{~A}$ \\
\hline 2000 & $13.00 \mathrm{e}$ & $15.77 \mathrm{c}$ & $16.53 b$ & $15.10 \mathrm{~B}$ & $5.73 \mathrm{~g}$ & $6.57 \mathrm{de}$ & $7.87 \mathrm{~b}$ & $6.72 \mathrm{~B}$ \\
\hline 4000 & $11.27 \mathrm{~g}$ & $13.87 \mathrm{~d}$ & $13.97 \mathrm{~d}$ & $13.03 \mathrm{C}$ & $5.00 \mathrm{~h}$ & $5.80 f g$ & $6.70 \mathrm{~cd}$ & $5.83 \mathrm{C}$ \\
\hline 6000 & $9.80 \mathrm{~h}$ & $11.70 \mathrm{fg}$ & $12.10 \mathrm{f}$ & 11.20D & $4.37 \mathrm{i}$ & $4.90 \mathrm{~h}$ & $5.87 \mathrm{fg}$ & 5.04D \\
\hline 8000 & $8.07 \mathrm{i}$ & $9.77 \mathrm{~h}$ & $10.10 \mathrm{~h}$ & $9.31 \mathrm{E}$ & $3.57 \mathrm{j}$ & $4.13 \mathrm{j}$ & $4.83 \mathrm{~h}$ & $4.18 \mathrm{E}$ \\
\hline \multirow[t]{2}{*}{ Mean } & $11.22 \mathrm{C}$ & 13.58B & $14.05 \mathrm{~A}$ & - & $4.97 \mathrm{C}$ & $5.69 \mathrm{~B}$ & $6.73 \mathrm{~A}$ & - \\
\hline & \multicolumn{8}{|c|}{ Second season 2019} \\
\hline 0.00 & $5.40 \mathrm{e}$ & $6.47 \mathrm{~cd}$ & $9.53 a$ & $7.13 \mathrm{~A}$ & $3.43 \mathrm{~d}-\mathrm{f}$ & $3.70 b-d$ & $4.53 \mathrm{a}$ & $3.89 \mathrm{~A}$ \\
\hline 2000 & $4.90 \mathrm{ef}$ & $6.07 \mathrm{~d}$ & $8.37 \mathrm{~b}$ & $3.45 \mathrm{~B}$ & $3.07 \mathrm{gh}$ & $3.60 \mathrm{c}-\mathrm{e}$ & $3.90 \mathrm{~b}$ & $3.52 \mathrm{~B}$ \\
\hline 4000 & 4.70ef & 5.80de & $7.88 \mathrm{bc}$ & $6.13 \mathrm{C}$ & $2.83 \mathrm{hi}$ & $3.33 \mathrm{e}-\mathrm{g}$ & $3.73 b c$ & $3.30 \mathrm{C}$ \\
\hline 6000 & $4.50 \mathrm{f}$ & $5.60 \mathrm{de}$ & $7.03 c$ & $5.71 \mathrm{D}$ & $2.70 \mathrm{ij}$ & $3.10 \mathrm{gh}$ & $3.30 \mathrm{fg}$ & $3.03 \mathrm{D}$ \\
\hline 8000 & $4.20 \mathrm{~g}$ & $5.01 \mathrm{e}$ & $6.40 \mathrm{~cd}$ & $5.20 \mathrm{E}$ & $2.43 \mathrm{j}$ & $2.87 \mathrm{hi}$ & $3.00 \mathrm{~h}$ & $2.77 \mathrm{E}$ \\
\hline Mean & $4.74 \mathrm{C}$ & $5.79 \mathrm{~B}$ & $7.84 \mathrm{~A}$ & - & $2.89 \mathrm{C}$ & $3.32 \mathrm{~B}$ & $3.69 \mathrm{~A}$ & - \\
\hline
\end{tabular}

Mean followed by the same letter in a column or row do not differ significantly according to Duncan's new multiple range test

\section{Chemical composition of the leaves}

As shown in Table 8, concentrations of chlorophyll a, b and carotenoids (mg/g. f. w.) and the percentages of $\mathrm{N}, \mathrm{P}$ and $\mathrm{K}$ exhibited a gradual decrease as a result of the progressive increment in salinity level, whereas $\mathrm{Na}$ and $\mathrm{Cl}$ percentages and proline 
(mg/100 g d. w.) were linearly increased. On the other hand, drenching the soil mixture with magnetite successively raised chlorophyll $\mathrm{a}, \mathrm{b}, \mathrm{N}, \mathrm{P}$ and $\mathrm{K}$ concentrations as the rate of magnetite was increased, but concentrations of carotenoids, $\mathrm{Na}, \mathrm{Cl}$ and proline were cumulatively declined. Generally, the combining between salinity level up to 4000 ppm and magnetic iron at the rate of $4 \mathrm{~g} /$ plant recorded the utmost high concentrations of chlorophyll a, b, N, P and $\mathrm{K}$, but that was true for carotenoids when plants grown in no salinized soil mixture and abandoned of $\mathrm{Fe}_{3} \mathrm{O}_{4}$, and for $\mathrm{Na}, \mathrm{Cl}$ and proline constituents when plants cultured in soil mixture which received 8000 ppm salinity level in the absence of $\mathrm{Fe}_{3} \mathrm{O}_{4}$.

The results pronouncedly clear that magnetite application markedly reduced $\mathrm{Na}^{+}$and $\mathrm{Cl}^{-}$toxic ions leading to mitigating their deleterious effects on growth and performance of Chinese hibiscus plants. As $\mathrm{Na}$ and $\mathrm{Cl}$ concentration was decreased by raising $\mathrm{Fe}_{3} \mathrm{O}_{4}$ rate the concentration of proline was diminished due to alleviating salt stress.

Table 7. Effect of salinity levels, magnetite rates and their interactions on resistance index and benefit coefficient of Hibiscus rosa-sinensis L. plant during 2018 and 2019 seasons

\begin{tabular}{|c|c|c|c|c|c|c|c|c|}
\hline \multirow{2}{*}{$\begin{array}{r}\mathrm{Fe}_{3} \mathrm{O}_{4} \text { rate } \\
(\mathrm{g} / \mathrm{plant})\end{array}$} & \multicolumn{4}{|c|}{ Resistance index (\%) } & \multicolumn{4}{|c|}{ Benefit coefficient of $\mathrm{Fe}_{3} \mathrm{O}_{4}$} \\
\hline & 0.00 & $2 \mathrm{~g}$ & $4 \mathrm{~g}$ & Mean & 0.00 & $2 \mathrm{~g}$ & $4 \mathrm{~g}$ & Mean \\
\hline & \multicolumn{8}{|c|}{ First season 2018} \\
\hline 0.00 & $100.00 \mathrm{a}$ & $100.00 \mathrm{a}$ & $100.00 \mathrm{a}$ & $100.00 \mathrm{~A}$ & $0.00 \mathrm{~g}$ & $11.41 \mathrm{e}$ & $40.50 \mathrm{~b}$ & 17.80B \\
\hline 2000 & $96.33 b$ & $96.28 b$ & $94.69 b$ & $95.76 \mathrm{~B}$ & $0.00 \mathrm{~g}$ & $13.48 \mathrm{~d}$ & $33.93 \mathrm{c}$ & $15.51 \mathrm{C}$ \\
\hline 4000 & $83.44 \mathrm{c}$ & $84.25 \mathrm{c}$ & $84.18 \mathrm{c}$ & $83.96 \mathrm{C}$ & $0.00 \mathrm{~g}$ & $7.83 \mathrm{f}$ & $33.59 \mathrm{c}$ & $13.81 \mathrm{D}$ \\
\hline 6000 & $73.24 d$ & $72.78 \mathrm{de}$ & $70.40 \mathrm{e}$ & $72.14 \mathrm{D}$ & $0.00 \mathrm{~g}$ & $11.88 \mathrm{de}$ & $39.84 b$ & 17.24B \\
\hline 8000 & $59.16 f$ & $59.43 f$ & $57.75 f$ & $58.78 \mathrm{E}$ & $0.00 \mathrm{~g}$ & $12.02 \mathrm{de}$ & $47.38 \mathrm{a}$ & $19.80 \mathrm{~A}$ \\
\hline \multirow[t]{2}{*}{ Mean } & $82.43 \mathrm{AB}$ & $82.55 \mathrm{~A}$ & $81.40 \mathrm{~B}$ & - & $0.00 \mathrm{C}$ & 11.32B & $39.05 \mathrm{~A}$ & - \\
\hline & \multicolumn{8}{|c|}{ Second season 2019} \\
\hline 0.00 & $100.00 \mathrm{a}$ & $100.00 \mathrm{a}$ & $100.00 \mathrm{a}$ & $100.00 \mathrm{~A}$ & $0.00 \mathrm{~g}$ & $12.15 \mathrm{~h}$ & $43.90 \mathrm{~d}$ & $18.68 \mathrm{C}$ \\
\hline 2000 & $66.60 \mathrm{~h}$ & $93.22 b$ & $87.70 \mathrm{~cd}$ & $82.51 \mathrm{C}$ & $0.00 \mathrm{~g}$ & $13.93 \mathrm{gh}$ & $42.84 d$ & $18.92 \mathrm{C}$ \\
\hline 4000 & $86.88 \mathrm{~cd}$ & $88.38 \mathrm{c}$ & $82.54 \mathrm{e}$ & $85.93 \mathrm{~B}$ & $0.00 \mathrm{~g}$ & $15.38 \mathrm{~g}$ & $47.68 \mathrm{c}$ & $21.02 \mathrm{~B}$ \\
\hline 6000 & $83.24 \mathrm{e}$ & $84.74 \mathrm{de}$ & $77.13 \mathrm{f}$ & $81.71 \mathrm{C}$ & $0.00 \mathrm{~g}$ & $19.71 \mathrm{f}$ & $59.78 \mathrm{a}$ & $26.50 \mathrm{~A}$ \\
\hline 8000 & $78.16 \mathrm{f}$ & $76.55 f$ & $70.71 \mathrm{~g}$ & $75.14 \mathrm{D}$ & $0.00 \mathrm{~g}$ & $24.51 \mathrm{e}$ & $55.94 b$ & $26.82 \mathrm{~A}$ \\
\hline Mean & 83.97B & $88.58 \mathrm{~A}$ & $83.62 \mathrm{~B}$ & - & $0.00 \mathrm{C}$ & 17.14B & $50.03 \mathrm{~A}$ & - \\
\hline
\end{tabular}

Mean followed by the same letter in a column or row do not differ significantly according to Duncan's New Multiple Range test

\section{Discussion}

\section{Effect of soil salinity, magnetite and their interaction on vegetative and root growth parameters}

From the previous results it was notice that the adverse effects of saline soil on growth may be attributed to either low water uptake due to low potential of soil water (Munns, 2002) or certain ion toxicity $\left(\mathrm{Na}^{+}\right.$and $\left.\mathrm{Cl}^{-}\right)$or both (Carter et al., 2005). This fact was emphasized by Elhag and Abdalla (2014) who stated that salinity depresses plant growth via affecting water absorption and biochemical processes such as $\mathrm{N}, \mathrm{CO}_{2}$ assimilation and protein biosynthesis or accumulated high concentration of potentially toxic ions $\left(\mathrm{Na}^{+}\right.$and $\left.\mathrm{Cl}^{-}\right)$. Jose et al. (2016) ascribed the reduction in growth by salinity 
to the effect of osmotic stress and the inhibition of cell division rather than cell expansion coupled with the great reduction in photosynthesis. High salinity also leads to leaf abscission due to ion accumulation in the leaves, particularly the oldest ones. Reduction of growth by salinity may be attributed to a decrease in all volume at a constant cell number. Mechanism of salt may result in inhibition of cell division, consequently reduces the rate of plant development (Khan et al., 2009). Jou et al. (2006), however suggested that ATPase participates in the endoplasmic reticulum-Golgi mediated, protein sorting machinery for both housekeeping function and compartmentalization of excess $\mathrm{Na}^{+}$under high salinity.

Table 8. Effect of salinity levels, magnetite rates and their interactions on some constituent's concentrations in the leaves of Hibiscus rosa-sinensis L. plants during 2019 seasons

\begin{tabular}{|c|c|c|c|c|c|c|c|c|c|c|c|c|}
\hline \multirow{2}{*}{$\begin{array}{r}\text { Fe3O4 rate } \\
\text { (g/plant) } \\
\text { Salinity level (ppm) }\end{array}$} & \multicolumn{4}{|c|}{ Chlorophyll a (mg/g f.w.) } & \multicolumn{4}{|c|}{ Chlorophyll b (mg/g f.w.) } & \multicolumn{4}{|c|}{ Carotenoids (mg/g f.w.) } \\
\hline & 0.00 & $2 \mathrm{~g}$ & $4 \mathrm{~g}$ & Mean & 0.00 & $2 \mathrm{~g}$ & $4 \mathrm{~g}$ & Mean & 0.00 & $2 \mathrm{~g}$ & $4 \mathrm{~g}$ & Mean \\
\hline & \multicolumn{12}{|c|}{ First season 2018} \\
\hline 0.00 & $2.320 \mathrm{~d}$ & $2.673 b$ & $3.040 \mathrm{a}$ & $2.678 \mathrm{~A}$ & $0.887 \mathrm{~cd}$ & $1.006 \mathrm{~b}$ & $1.163 \mathrm{a}$ & $1.019 \mathrm{~A}$ & $0.819 \mathrm{a}$ & $0.533 \mathrm{c}$ & $0.537 \mathrm{c}$ & $0.630 \mathrm{~A}$ \\
\hline 2000 & $1.765 \mathrm{~h}$ & $2.135 \mathrm{f}$ & $2.669 \mathrm{~b}$ & 2.190B & $0.794 \mathrm{ef}$ & $0.845 \mathrm{de}$ & $1.056 \mathrm{~b}$ & $0.898 \mathrm{~B}$ & $0.658 \mathrm{~b}$ & $0.494 d$ & $0.461 \mathrm{e}$ & $0.538 \mathrm{~B}$ \\
\hline 4000 & $1.512 \mathrm{j}$ & $2.258 \mathrm{e}$ & $2.605 \mathrm{c}$ & $2.125 \mathrm{C}$ & $0.687 \mathrm{~g}$ & $0.799 \mathrm{ef}$ & $0.932 \mathrm{c}$ & $0.806 \mathrm{C}$ & $0.447 \mathrm{e}$ & $0.410 \mathrm{fg}$ & $0.418 \mathrm{f}$ & $0.425 \mathrm{C}$ \\
\hline 6000 & $1.450 \mathrm{c}$ & $1.610 \mathrm{i}$ & $1.821 \mathrm{i}$ & $1.627 \mathrm{D}$ & $0.689 \mathrm{~g}$ & $0.743 \mathrm{fg}$ & $0.940 \mathrm{c}$ & $0.791 \mathrm{C}$ & $0.450 \mathrm{e}$ & $0.381 \mathrm{gh}$ & $0.394 \mathrm{fg}$ & $0.408 \mathrm{D}$ \\
\hline 8000 & $0.675 n$ & $0.811 \mathrm{~m}$ & 1.0621 & $0.849 \mathrm{E}$ & $0.553 \mathrm{~h}$ & $0.680 \mathrm{~g}$ & $0.710 \mathrm{~g}$ & $0.648 \mathrm{D}$ & $0.387 \mathrm{gh}$ & $0.359 \mathrm{~h}$ & $0.380 \mathrm{gh}$ & $0.375 \mathrm{E}$ \\
\hline \multirow[t]{2}{*}{ Mean } & $1.545 \mathrm{C}$ & $1.898 \mathrm{~B}$ & $2.239 \mathrm{~A}$ & - & $0.722 \mathrm{C}$ & $0.815 \mathrm{~B}$ & $0.960 \mathrm{~A}$ & - & $0.552 \mathrm{~A}$ & $0.436 \mathrm{~B}$ & $0.438 \mathrm{~B}$ & - \\
\hline & \multicolumn{4}{|c|}{$\mathrm{N}(\%)$} & \multicolumn{4}{|c|}{$\mathrm{P}(\%)$} & \multicolumn{4}{|c|}{$\mathrm{K}(\%)$} \\
\hline & 0.00 & $2 \mathrm{~g}$ & $4 \mathrm{~g}$ & Mean & 0.00 & $2 \mathrm{~g}$ & $4 \mathrm{~g}$ & Mean & 0.00 & $2 \mathrm{~g}$ & $4 \mathrm{~g}$ & Mean \\
\hline 0.00 & $3.475 \mathrm{~d}$ & $3.861 \mathrm{~b}$ & $4.147 \mathrm{a}$ & $3.828 \mathrm{~A}$ & $0.829 \mathrm{a}$ & $0.843 \mathrm{a}$ & $0.854 \mathrm{a}$ & $0.842 \mathrm{~A}$ & $1.696 \mathrm{e}$ & $1.983 \mathrm{~b}$ & $2.012 \mathrm{a}$ & $1.897 \mathrm{~A}$ \\
\hline 2000 & $3.088 \mathrm{~g}$ & $3.187 \mathrm{e}$ & $3.501 \mathrm{c}$ & $3.259 \mathrm{~B}$ & $0.753 b$ & $0.758 \mathrm{~b}$ & $0.775 \mathrm{~b}$ & $0.762 \mathrm{~B}$ & $1.552 \mathrm{f}$ & $1.753 \mathrm{~d}$ & $1.892 \mathrm{c}$ & $1.732 \mathrm{~B}$ \\
\hline 4000 & $2.779 \mathrm{i}$ & $2.891 \mathrm{~h}$ & $3.153 \mathrm{f}$ & $2.941 \mathrm{C}$ & $0.599 \mathrm{~d}$ & $0.671 \mathrm{c}$ & $0.698 \mathrm{c}$ & $0.656 \mathrm{C}$ & $1.208 \mathrm{j}$ & $1.394 \mathrm{~h}$ & $1.534 \mathrm{~g}$ & $1.380 \mathrm{C}$ \\
\hline 6000 & 2.3031 & $2.376 \mathrm{k}$ & $2.575 \mathrm{j}$ & $2.418 \mathrm{D}$ & $0.493 \mathrm{e}$ & $0.550 \mathrm{~d}$ & $0.563 \mathrm{~d}$ & $0.535 \mathrm{D}$ & $1.087 \mathrm{k}$ & $1.223 \mathrm{j}$ & $1.350 \mathrm{i}$ & $1.220 \mathrm{E}$ \\
\hline 8000 & $1.843 \mathrm{o}$ & $1.873 n$ & $1.991 \mathrm{~m}$ & $1.902 \mathrm{E}$ & $0.392 \mathrm{f}$ & $0.456 \mathrm{e}$ & $0.457 \mathrm{e}$ & $0.435 \mathrm{E}$ & $1.879 \mathrm{c}$ & 0.9801 & $1.080 \mathrm{k}$ & $1.313 \mathrm{D}$ \\
\hline \multirow[t]{3}{*}{ Mean } & $2.698 \mathrm{C}$ & $2.838 \mathrm{~B}$ & $3.073 \mathrm{~A}$ & - & $0.613 \mathrm{~B}$ & $0.656 \mathrm{~A}$ & $0.669 \mathrm{~A}$ & - & $1.484 \mathrm{~B}$ & $1.467 \mathrm{C}$ & $1.574 \mathrm{~A}$ & - \\
\hline & \multicolumn{4}{|c|}{$\mathrm{Na}(\%)$} & \multicolumn{4}{|c|}{$\mathrm{Cl}(\%)$} & \multicolumn{4}{|c|}{ Proline (mg/100 g f.w.) } \\
\hline & 0.00 & $2 \mathrm{~g}$ & $4 \mathrm{~g}$ & Mean & 0.00 & $2 \mathrm{~g}$ & $4 \mathrm{~g}$ & Mean & 0.00 & $2 \mathrm{~g}$ & $4 \mathrm{~g}$ & Mean \\
\hline 0.00 & $0.812 \mathrm{~h}$ & $0.796 \mathrm{~h}$ & $0.730 \mathrm{i}$ & $0.779 \mathrm{E}$ & $1.460 \mathrm{~h}$ & $1.460 \mathrm{~h}$ & $1.490 \mathrm{~h}$ & $1.470 \mathrm{E}$ & $53.23 \mathrm{k}$ & 49.121 & $45.48 \mathrm{~m}$ & $49.27 \mathrm{E}$ \\
\hline 2000 & $0.933 \mathrm{~g}$ & $0.914 \mathrm{~g}$ & $0.813 \mathrm{~h}$ & $0.887 \mathrm{D}$ & $1.780 \mathrm{f}$ & $1.670 \mathrm{~g}$ & $1.500 \mathrm{~h}$ & $1.650 \mathrm{D}$ & $68.16 \mathrm{~h}$ & $64.83 i$ & $64.77 \mathrm{i}$ & $65.92 \mathrm{D}$ \\
\hline 4000 & $1.120 \mathrm{f}$ & $1.118 \mathrm{f}$ & $1.108 \mathrm{f}$ & $1.115 \mathrm{C}$ & $2.050 \mathrm{~d}$ & $1.830 \mathrm{f}$ & $1.620 \mathrm{~g}$ & $1.830 \mathrm{C}$ & $74.32 \mathrm{~g}$ & $65.35 \mathrm{i}$ & $62.49 j$ & $67.39 \mathrm{C}$ \\
\hline 6000 & $1.543 b$ & $1.416 \mathrm{~d}$ & $1.133 \mathrm{ef}$ & $1.364 \mathrm{~B}$ & $2.360 \mathrm{~b}$ & $2.050 \mathrm{~d}$ & $1.830 \mathrm{f}$ & $2.080 \mathrm{~B}$ & $89.19 \mathrm{~d}$ & $79.73 \mathrm{e}$ & $76.36 \mathrm{f}$ & $81.76 \mathrm{~B}$ \\
\hline 8000 & $1.850 \mathrm{a}$ & $1.481 \mathrm{c}$ & $1.154 \mathrm{e}$ & $1.495 \mathrm{~A}$ & $2.590 \mathrm{a}$ & $2.230 \mathrm{c}$ & $1.950 \mathrm{e}$ & $2.260 \mathrm{~A}$ & $129.50 \mathrm{a}$ & $113.61 \mathrm{~b}$ & $99.91 \mathrm{c}$ & $114.34 \mathrm{~A}$ \\
\hline Mean & $1.252 \mathrm{~A}$ & $1.145 \mathrm{~B}$ & $0.988 \mathrm{C}$ & - & $2.050 \mathrm{~A}$ & $1.850 \mathrm{~B}$ & $1.680 \mathrm{C}$ & - & $82.88 \mathrm{~A}$ & 74.53B & $69.80 \mathrm{C}$ & - \\
\hline
\end{tabular}

Means followed by the same letter is a column or row do not differ significantly according to Duncan's new multiple range test

Likewise, Cassaniti et al. (2013) claimed that brackish water can be commercially used for production of Chrysanthemum morifolium, Dianthus caryophyllus, Gerbera jamesonii, Hippeastrum vittatum and Anthurium andreanum, but saline water higher than $2500 \mathrm{ppm}$ concentration reduced their growth, delayed flowering and decreased flower quality. Similarly, were those results of Shahin et al. (2008) on Ficus macrocarpa var. Hawaii and Euonymus Japonica cv. Mediopicta, Shahin et al. (2013) on Ficus benjamina cv. Samantha and Schefflera arboricola cv. Gold Capella, El-Fouly et al. (2015) on Iris tingitana cv. Wedgewood, Jose et al. (2016) on Eucalyptus urophylla and the hybrid of E. urophylla $\mathrm{x}$ E. grandis.

On the other hand, the positive effect of magnetic iron on growth of plants suffered from salt stress may be due to its role in promoting the uptake of $\mathrm{N}, \mathrm{P}, \mathrm{K}$ and Fe which 
stimulate plant growth against the toxicity of $\mathrm{Na}^{+}$and $\mathrm{Cl}^{-}$ions that inhibit it. It induces cell metabolism and mitosis of meristematic cells (Barage et al., 2009). It is believed that new protein bands are formed in plants that are treated with $\mathrm{Fe}_{3} \mathrm{O}_{4}$ and these proteins are responsible for the increased growth (Hozyan and Abdul-Qodos, 2010). Furthermore, it declines the hydration of salt ions and colloids, increasing salt solubility, and finally leading to leaching such salts from the soil. So, it is successfully used to reclaim soils with high cations and anions, such as $\mathrm{Ca}, \mathrm{Na}, \mathrm{Cl}$ and $\mathrm{HCO}_{3}$ (Mostafazadeh et al., 2012). In the iron atom, there is a number of valence electrons that generates a magnetic field influence on the biochemical processes in plants and renders the roots to exhibit symptoms of magnetism that kills nematodes and injurious bacteria (Yuliando et al., 2016). Supporting results for those of this study were also obtained by Abdel Fattah (2014) on Jacaranda ocutifolia, Ahmed et al. (2016) on Acalypha wilkesiana, Shahin et al. (2018) on Terminalia arjuna and El-Sayed et al. (2019) on elephant`s ear tree.

\section{Salt resistance index (SRI\%) and benefit coefficient of magnetite (B. coe.\%)}

Such gains may be reasonable, as they indicate the role of magnetic in alleviating salt hazards and promoting dry matter production. These results are reasonable because magnetite usually solubilizes and leaches salts from the soil, and this gives roots a chance to penetrate and distribute well through the growing medium, consequently uptake enough water and nutrients necessary for good and healthy growth. On the same line were those results attained by Al-Qubaie et al. (2003) on Ficus benghalensis, Bougainvillea glabra, Jasminum azoricum, Conocarpus erectus, Tamarix articulata and Ziziphus spinachristi, Shahin et al. (2008) on Ficus macrocarpa var. Hawaii and Euonymus Japonica cv. Mediopicta, Abdel-Fattah et al. (2012a) on Ficus retusa, Abdel Fattah et al. (2012b) on Ficus benjamina and El-Sayed et al. (2019) Enterolobium contortisiliquum.

\section{Chemical composition of the leaves}

This may be reasonable because salts usually reduces the water potential of soil solution, consequently decrease minerals and water uptake by roots, accompanied by a depression of photosynthesis and enzymes activity (Munns, 2002). It was also remarkable that accumulation of some amino acids and amides in the leaves and roots of salinity-stressed plants may be attributed to de novo synthesis by protein sorting machinery and not the result of protein degradation (Jou et al., 2006).

These results, are similar trend to those of Elhag and Abdalla (2014) and Moustafa et al. (2017) on Moringa oleifera, Ahmed et al. (2016) on Acalypha wilkesiana, Shahin et al. (2018) on Terminalia arjuna and El-Sayed et al. (2019) who observed that chlorophyll a, b, carotenoids and total carbohydrates concentrations were increased in the leaves of Enterolobium contortisiliquum under salt stress by application of $\mathrm{Fe}_{3} \mathrm{O}_{4}$ at either 2 or $4 \mathrm{~g} /$ pot.

\section{Conclusion}

Hence, it can be recommended to drench the soil mixture, in which hibiscus of China plants are grown with $4 \mathrm{~g} /$ pot of magnetic iron $\left(\mathrm{Fe}_{3} \mathrm{O}_{4}\right)$ to improve their growth and aesthetic value under salt stress during rearing in the nursery.

It is recommended to use transplants of different ages growing under higher dose of salinity. 


\section{REFERENCES}

[1] Abdel-Fattah, G. H., Shahin, S. M., Khenizy, S. A. M. (2012a). Improving tolerance of Ficus benjamina L. plant to saline water stress by VAM (Glomus sp.). - J. Biol. Chem. \& Environ. Sci. 7(1): 1-16.

[2] Abdel-Fattah, G. H. (2014): The role of magnetic iron and sodium salenate in minimizing soil salt hazards on growth and quality of Jacaranda acutifolia Humb. \& Bionol seedlings. - Sci. J. Flowers \& Ornam. Plants 1(3): 187-198.

[3] Abdel-Fattah, G. H., Shahin, S. M., Abdel-Moniem, A. M. (2012b). Could phosphorene help Ficus retosa L. plant to tolerate the deleterious effects of saline water? - J. Biol. Chem. \& Environ. Sci. 7(1): 17-32.

[4] Ahmed, M., A. (2017): Effect of salinity $\left(\mathrm{NaCl}+\mathrm{CaCl}_{2}\right)$ and some soil additions on growth, flowering and chemical composition of rose of China (Hibiscus rosa-sinensis L.) plant. - Middle East J. Agric. Res. 6(2): 302-314.

[5] Ahmed, Y. M., Shalaby, E. A., Shanan, N. T. (2011): The use of organic and inorganic cultures in improving vegetative growth, yield characters and antioxidant activity of roselle plants (Hibiscus sabdariffa L.). - African J. Biotech.,10(11): 1988-1996.

[6] Ahmed, M., A., Abdel-Fattah, G. H., Shahin, S. M. (2016): The role of magnetic iron in enhancing the ability of Acalypha wilkesiana Mull. Arg. transplants to tolerate soil salinity. - J. Plant Production, Mansoura Univ. 7(3): 379-384.

[7] Al-Qubaie, A. I., Shahin, S. M., Abdel-Samad, F. A. (2003): Effect of diluted sea water on growth and chemical composition of some ornamental trees and shrubs. - Egypt J. Appl. Sci. 18(8 B): 587-601.

[8] Bailey, L. H. (1976): Hortus. - Macmillan Publishing Co., Inc., New York.

[9] Barage, L. F., da Sousa, M. P., Almeida, T. A. (2009): Enterolobium schomburgkii Benth. Seed germination under saline stress and polyamine application. - Revista Braz Plantas Med. 11(1): 1516-1527.

[10] Batels, L. S., Walden, R. P., Teare, T. D. (1973): Rapid determination of free proline under water stress studies. - Plant and Soil 939: 205-207.

[11] Blake, C. A. (1965): Methods of Soil Analysis Part 1 Physical and Mineralogical Properties Including a Statistics of Measurement and Sampling. - Amr. Soc. Agron. Inc. Pub., Madison, WI.

[12] Carter, C. T., Grieve, C. M., Poss, J. A. (2005): Salinity effects on emergence, survival and ion accumulation of Limonium perezii. - J. Plant Nutrition 28: 1243-1257.

[13] Cassniti, C., Romano, D., Hop, M. E., Flowers, T. J. (2013): Growing floricultural crops with brackish water. - Environmental and Experimental Botany 92: 165-175.

[14] El-Fouly, A. S., Abdel-Sattar, M. M., Shahin, S. M. (2015): Effect of different salinity levels, PP-333 treatments and their interaction on growth, flowering, bulbs productivity and chemical composition of Iris tingitana cv. Wedgewood. - J. Agric. Res. Kafr ElSheikh Univ. 41(1): 347-369.

[15] Elhag, A. Z., Abdalla, M. H. (2014): Investigation of sodium chloride tolerance of Moringa oleifera Lam. transplants. - Universal J. Agric. Res. 2(2): 45-49.

[16] El-Sayed, B. A., Shahin, S. M., El-Tayeb, H. F. (2015): Response of saltbush (Atriplex halimus L.) plants to salinity and type of growing media. - Scientific J. Flowers \& Ornam. Plants 2(1): 135-148.

[17] El-Sayed, M. A., El-Fouly, Amal, S., A., Shahin, S. M. (2019): Reducing the harmful effect of saline irrigation water on growth and quality of Enterolobium contortisiliquum (Elephant`s ear) seedlings. - Bull, Fac. Agric. Cairo. Univ. 70: 267-276.

[18] El-Shewaikh, Y. M. E., Khenizy, S. A. M., El-Deeb, E. E. A. (2015): Effect of some physiological treatments on areca seedlings. B. Effect of irrigation with saline water on areca (Dypsis lutescens Wendl.) seedlings. - J. Biol, Chem., \& Environ. Sci. 10(4): $297-$ 308. 
[19] Hozyan, M., Abdul-Qodos, A. (2010): Irrigation with magnetized water enhances growth, chemical constituent and yield of chickpea (Cicer arietinum L.). - Agric. and Biol. J. of Amer. 1(4): 671-676.

[20] Jackson, M. H. (1973): Soil Chemical Analysis. - Prentice-Hall of India Private Limited, New Delhi.

[21] Jose, A. C., Silva, N. C. N., Faria, J. M., Pereira, W. V. (2016): Influence of osmotic conditioning of Eucalyptus spp. seeds in the tolerance to salt stress. - J. Seed Sci. 38(4): 329-334.

[22] Jou, Y., Chiang, C., Jauh, G., Yen, H. (2006): Functional characterization of ice plant, on AAA-type ATPase associated with the endoplasmic reticulum-Golgi network, and its role in adaptation to salt stress. - Plant Physiol. 141(1): 135-146.

[23] Khafagy, M. A., Abdalla, M. Y. A., Hussein, H. A. A., Ahmed, S. A. M. (2013): Response of Hibiscus rosa-sinensis L. to the interactive effect of seawater salinity and ascorbic acid. - J. Plant Production Mansoura Univ. 4(1): 51-78.

[24] Khan, G. S., Khan, Z. H., Imran, J., Quraishi, M. U., Yaqoob, S., Khan, S. H. (2009): Effect of salinity on germination and growth of some forest tree species at seedling growth. - J. Agric. Res. 47(3): 271-279.

[25] Kratsch, H., Olsen, S., Rupp, L., Cardon, G., Heflebower, R. (2008): Soil Salinity and Ornamental Plant Selection. - Horticulture Utah State Univ., Cooperative Extension, Logan, pp. 2-8.

[26] Luatanab, F. S., Olsen, S. R. (1965): Test of an ascorbic acid method for determining phosphorus in water and $\mathrm{NaHCO}_{3}$ extracts from soil. - Soil Sci. Soc. Amer. Proc. 29: 677-678.

[27] Mahmoud, A. M. A., Ahmed, S. S., El-Feky, A. H. (2008): Effect of diluted sea water on growth and chemical composition of two ornamental hedges grown in some soils of Egypt. - J. Agric. Res. Kafr El-Sheikh Univ. 34(2): 522-535.

[28] Mcfarland, M. L., Provin, T. L., Redmon, L. A., Boellstorff, D. E., McDonald, A. K., Stem, L. A., Wherley, B. G. (2014): An Index of Salinity and Boron Tolerance of Common Native and Introduced Plant Species in Texas. - Texas A \& M AgriLife Extension Service, College Station, Texas.

[29] Mead, R., Curnow, R. N., Harted, A. M. (1993): Statistical Methods in Agriculture and Experimental Biology. $2^{\text {nd }}$ Ed. - Chapman \& Hall Ltd., London.

[30] Mostafazadeh, B., Khoshrovesh, M., Mousove, S. F., Kiani, A. R. (2012): Effects of Magnetized Water on Soil Chemical Components Underneath Trick Irrigation. - Amer. Soc. of Civil Engineers. Reston, VA. http://dx.doi.org/10.1061/(ASCE) IR. 19434774.0000513 .

[31] Moustafa, H. E. B., Abdel-Fattah, G. H., Shahin, S. M. (2017): Germination of Moringa oleifera Lam seeds under salinity conditions in the presence of magnetic iron. - J. Biol. Chem. \& Environ. Sci. 12(3): 551-566.

[32] Munns, R. (2002): Comparative physiology of salt and water stress. - Plant Cell and Environment 25: 239-250.

[33] SAS Institute (2009): SAS/STAT User's Guides Statistics. Vers. 6.04, $4^{\text {th }}$ Ed. - SAS Institute Inc. Cary, N. C., USA.

[34] Shahin, S. M., Eliwa, Naglaa, Y., Mahmoud, A. M. A., El-Feky, A. H. (2008): How far diluted sea water can be used for irrigation of some pot plants. - Proc. $9^{\text {th }}$ Inter. Conf. on Dry Land Development, 7-10 Nov., Organized by IDDC, Alex., Egypt, pp. 363-372.

[35] Shahin, S. M., El-Fouly, A. S., Abdel-Sattar, M. M. (2013): Response of F. benjamina L. cv. Samantha and Schefflera arboricola Endl cv. Gold Copella plants cultivated in two different growing media to salinity of irrigation water. - J. Biol. Chem. \& Environ. Sci. 8(2): 201-218.

[36] Shahin, S. M., El-Sayed, B. A., El-Tayeb, H. F. (2014): Impact of irrigation with saline water on growth, quality and chemical composition of tall fescue turf (Festuca 
arundinacea Schreb. var. Festorina). - Scientific J. Flowers \& Ornam. Plants 1(2): 137144.

[37] Shahin, S. M., Ahmed, M. A., Tawila, A. S. (2017): Effect of saline irrigation water on growth and quality of Casuarina equistifolia L. and Eucalyptus rostrata Schlecht plants grown in some soils of Egypt. - Middle East J. Appl. Sci. 7(3): 460-471.

[38] Shahin, S. M., Aly, A. M., Helaly, A. A. E. (2018): Germination of Indian almond (Terminal arjuna Roxb.) seeds as affected by soil salinity in presence or absence of magnetic iron. - J. Plant Production, Mansoura Univ. 9(4): 417-422.

[39] Steel, R. G. D., Torrie, J. H. (1980): Principles and Procedures of Statistics. - McGraw Hill Book Co., Inc., New York, pp. 377-400.

[40] Sumanta, N., Haque, C. I., Nishika, J., Suprakash, R. (2014): Spectrophotometric analysis of chlorophyllous and carotenoids from commonly grown fern sp. by using various extracting solvents. - Research J. Chem. Sci. 4(9): 63-69.

[41] Wu, L., Huff, D. R. (1983): Characteristics of creeping bent grass clones (Agrostis stolonifera L.) from a salinity tolerant population after surviving drought stress. - Hort Science 18(6): 883-885.

[42] Yuliando, A., Astuti, B., Amalia, S. R. (2016): Enhancement of iron content in spinach plants stimulated by magnetic nano particles. - Proc. $3^{\text {rd }}$ Inter Conf. on Advanced Materials Science and Technology (ICAMST), 19 April, 2016. http//doi.org./10.1063/1.4945554. 